\title{
The Potential of Phase-Doppler Anemometry ${ }^{\dagger}$
}

\author{
Klaus Bauckhage \\ Stiftung Institut für Werkstofftechnik (IWT)*
}

\begin{abstract}
Phase-Doppler anemometry (PDA) is a well-established optical measuring technique for the nonintrusive and simultaneous determination of size and velocity of spherical particles in various engineering processes. The design of a phase-Doppler system includes simulations based on the Mie theory, which describe the scattering of a plane wave by a spherical particle. Although the greater number of particles in technical processes or in the natural environment are non-spherical, the real shape is neglected and the particles are assumed to be perfectly spherical, with smooth surfaces, and to consist of homogeneous materials. In practice, it was found that the size-response of a phaseDoppler system deviates from the theoretical prediction when these assumptions are not fulfilled. In this paper, optical arrangements and adaptations of theories of light scattering will be discussed which utilize these deviations to provide even more information on the nature of the particles being examined.
\end{abstract}

\section{Introduction}

The main field of successful PDA application has been established in the atomization of liquids in connection with heat and mass transfer processes. Research has focused mainly on spray analysis and droplet transport of pure liquids (e.g. water, fuels and organic liquids) in gaseous two-phase flows. Good progresses were made in this area not only in process automation, but also in high-precision on-line and inline measurements [1 to 6]. This is not surprising, since in most of these cases, the real shape of, e.g. water droplets in air, is indeed perfectly spherical, and the particulate material is homogeneous, thus fulfilling the main assumptions of the Mie theory.

On the other hand, adaptations of the optical set-up have to be provided for measuring droplets of opaque (absorbing) fluids, of metal melts, or (instead of pure liquids) droplets which contain inclusions - so-called optical inhomogeneities. Problems may also occur due to the surface roughness of solid particles. Therefore, it is in many cases helpful to use light scattering simulations based on geometrical optics (GO) and the Mie theory for the design and arrangement,

\footnotetext{
* Bremen, Germany

* Accepted: September 17, 2001
}

and finally for optimisation, of the optical set-up prior to taking measurements.

The assumption that PDA measurements from single particles may apply as absolute measures has to be given up when particle collectives such as sprays or powders need analysing, resulting in measured size distributions which often contain under-representations of the fine particle spectrum.

The purpose of this paper, after a short explanation of the standard PDA set-up and a brief discussion about the necessity of optimisation of PDA arrangements (against the background of small particles), is to outline the problems of inhomogeneous and deformed droplets and the rough surfaces of particles, because these give rise to a number of problems in spray analysis. Since the sizing errors of larger particles caused by the Gaussian beam effect of the intensity distribution of the laser beams are widely described and can be eliminated either by upgraded PDA arrangements or by special signal post-processing programs, this aspect needs no discussion here.

\section{Standard PDA set-up}

A PDA system in its most simple form is made up of two laser beams and two optical detectors. The schematic of such a standard PDA system is shown in Fig. 1. (Commercial PDA systems often employ a 
third or a fourth detector to allow cross-validation of the individual results.) The two laser beams intersect and define the optical probe volume, which can be interpreted as consisting of parallel planes of alternating light intensity resulting from interference of the laser beams. Particles passing this intersection volume scatter the light of both incident laser beams. Interference between the scattered light leads to the detection of so called bursts by two optical receivers (PM1 and PM2). The main frequency $f_{D}=1 / T$ of these bursts is a measure of the particle velocity component $v_{z}$, while a temporal delay between the signals $\Delta T$ (or phase-difference $\Delta \Phi$ ) can be related to particle diameter $d$ by means of appropriate light scattering computations. As long as individual particles moving through the probe volume are spherical, homogeneous and clearly larger than the laser wavelength $\lambda$ of the laser, the intensity of the scattered light is strong enough to be detected without any difficulty.

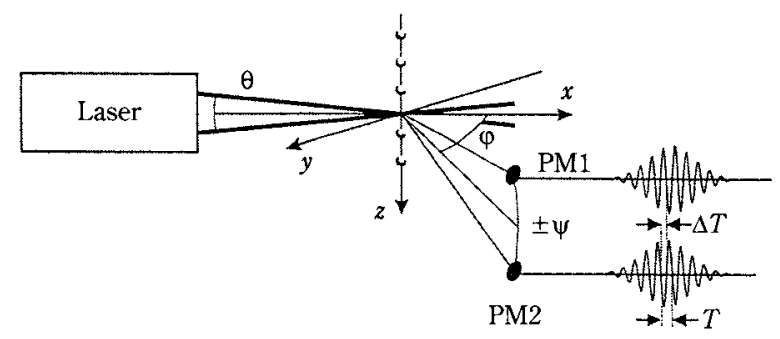

Fig. 1 Set-up of a standard phase-Doppler anemometer (with two optical receivers). Definition of beam-crossing angle $\theta$, elevation angles $\psi$, and off-axis angle $\varphi$. The phase-difference is defined as $\Delta \Phi=2 \pi \Delta T / T$
Furthermore, for defining an optimal arrangement of the set-up, a careful analysis of the performance characteristics of the instrumentation system with respect to the measuring task is required. This holds true for the positions of the detectors (the off-axis angle $\varphi$, and the elevation angles $\psi$ are the important parameters) and the beam configuration system (laser wavelength $\lambda$, power $P$, beam waist radius $\omega$, and beam-crossing angle $\phi$ ). These parameters should be chosen mainly according to the particulate material (i.e. the refractive index $m=n+k i$ ) and the individual size range of the particles. The relation between the measured phase-difference $\Delta \Phi$ and the particle diameter $d$ is linear as given in Equation (1) in Table 1. The exact relation can be obtained in a closed form from geometrical optics for solely reflecting (Eq. 2) or refracting (Eq. 3) particles as given in Table 1, where the relative refractive index (i.e. the ratio of the refractive indices of the dispersed (droplet) phase to the continuous (bulk) phase $n^{\prime}=n_{d} / n_{c}$ ) is given by Equation (4) [7 and 8].

\section{Optimisation of the PDA arrangement}

In order to determine the optimal arrangement of the two detectors to suit the measuring problem, one has to recall some fundamentals of light scattering by a spherical particle, starting with light scattering simulations based on geometrical optics (GO) and the Mie theory. Such simulation tools have been used, e.g. [9 and 10], plotting for instance polar diagrams, as given in Fig. 2 for a water droplet in air $(n=1.333$; $k=0)$ and a tin melt droplet in nitrogen $(n=2.100 ; k=$ 4.5). The laser beam (from the left) has a wavelength of $\lambda=0.632 \mu \mathrm{m}$ and the drop diameter is $d=50 \mu \mathrm{m}$. The radial axis of each diagram shows the intensity of

Table 1 Equations for the droplet diameter, based on geometrical optics

$d=\frac{1}{2 b}\left(\frac{\lambda}{\pi n_{\mathrm{c}}}\right) \Delta \Phi$
$b_{\text {Reflex }}=\sqrt{2}\left[\left(1+\sin \frac{\theta}{2} \sin \Psi-\cos \frac{\theta}{2} \cos \Psi \cos \varphi\right)^{\frac{1}{2}}-\right.$
$\left.-\left(1-\sin \frac{\theta}{2} \sin \Psi-\cos \frac{\theta}{2} \cos \Psi \cos \varphi\right)^{\frac{1}{2}}\right]$
$b_{\text {Brech. }}=2\left\{\left[1+n^{\prime 2}-\sqrt{2} n^{\prime}\left(1+\sin \frac{\theta}{2} \sin \Psi+\cos \frac{\theta}{2} \cos \Psi \cos \varphi\right)^{\frac{1}{2}}\right]^{\frac{1}{2}}-\right.$
$\left.\quad-\left[1+n^{\prime 2}-\sqrt{2} n^{\prime}\left(1-\sin \frac{\theta}{2} \sin \Psi+\cos \frac{\theta}{2} \cos \Psi \cos \varphi\right)^{\frac{1}{2}}\right]^{\frac{1}{2}}\right\}$
$n^{\prime}=\frac{n_{\mathrm{d}}}{n_{\mathrm{c}}}$


Pthesscattered light in a logarithmic scale versus the off-axis angle $\varphi$.

Mie calculations make no distinction between the different light components (the results are given in Fig. $2 \mathbf{a}$ as sectional views in two main planes of a hemisphere around the scattering particle in its centre). On the other hand, under geometrically optimal conditions, the light intensities may be differentiated, being plotted as either diffracted (Fig. 2b), refracted (Fig. 2c) or reflected (Fig. 2d) light components. (The lower part of the diagram holds true for parallel polarised light, the upper part for perpendicular polarisation). Particle sizing has to be executed out of the diffraction domain (off-axis angles $\varphi$ up to $10^{\circ}$ ), otherwise phase-differences do not appear.
The message from these plots (Fig. 2 a to $\mathbf{d}$ ) is that transparent (pure) liquids should be measured under off-axis angles $10^{\circ}<\varphi<60^{\circ}\left(70^{\circ}\right.$ for parallel polarised light) using the refraction domain.

In the case of non-transparent particles with an absorption factor $k>0$ of the refractive index, where the intensity of the refracted light portion is diminished and reflection becomes more and more dominant, the comprehensive simulation of light scattering becomes compulsory in order to find the best off-axis configuration for the set-up. This will be discussed in detail below, when the Gain/ $\varphi$-diagram [11 and 12] will be introduced.

Small droplets of molten metal are also spherical in shape because of their high surface tension. The high
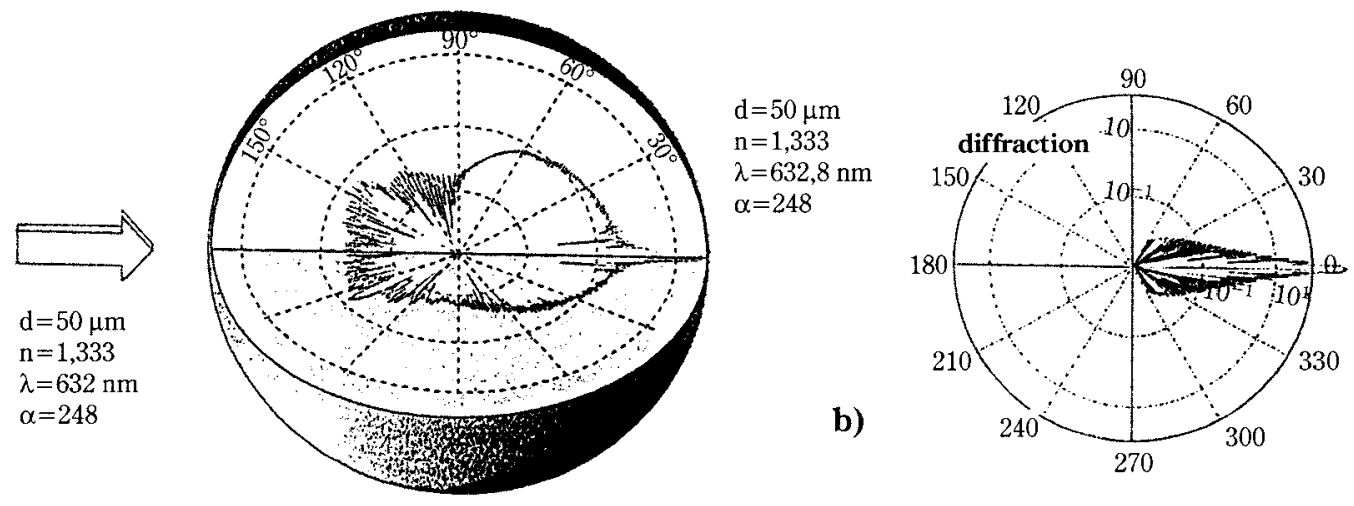

a)

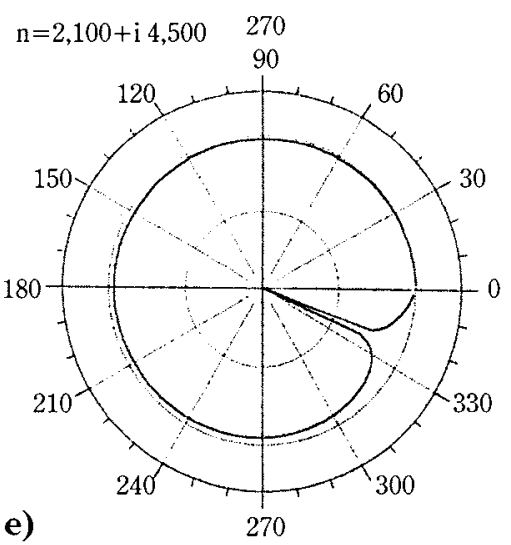

c)
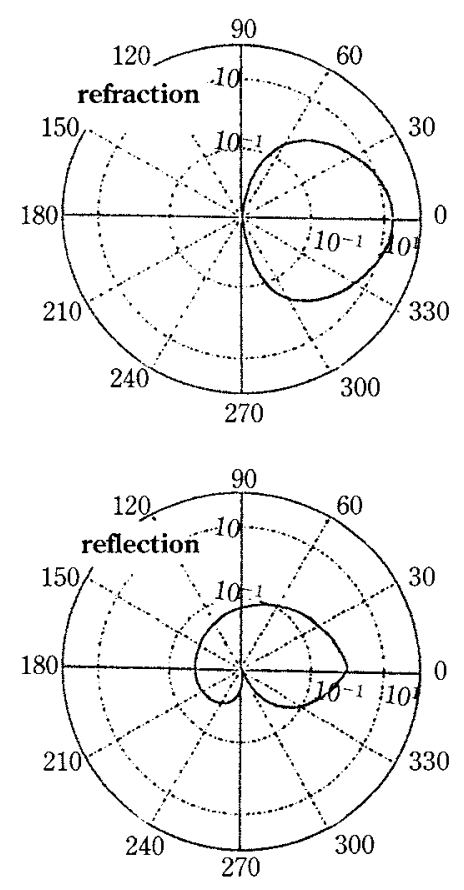

d)

Fig. 2 Results from Mie-calculations a) and from GO-calculations b) to e) for light scattering of a droplet in air a) to d) $\{n=1.333 ; k=0\}$ and a tin melt droplet in nitrogen e) $\{n=2.100 ; k=4.5\} d=50 \mu \mathrm{m} ; \lambda=0.632 \mu \mathrm{m}$ 
imaginary part of their refractive index (e.g. for a tin droplet: $n=2.100+4.5$ i) causes a strong and sole domain of reflection for all off-axis angles in the polar diagram (Fig. 2e). This holds true for angles $\varphi$ from $10^{\circ}$ to $180^{\circ}$, enlarging the arrangement facility of the PDA receivers significantly, and allowing observation of the particles, for instance, from a back-scatter position [13 to 15].

Polar diagrams can even be plotted to specify the liquid droplet scattering behaviour in liquid environments, e.g. describing details of liquid/liquid extraction processes, where the relative refractive index $\left(n^{\prime}=n_{d} / n_{c}\right)$ approximates values of 1 [16 to 18]. Such plots allow a decision to be made on whether PDA measurements make sense, and if so, from which angular position the droplets should be observed, and whether the droplets have to be treated as refracting or reflecting particles. It is worth mentioning that the off-axis angles from these computations generally have to be set equal to those off-axis angles of the PDA detectors in relation to the measuring volume, i.e. to the droplet to be measured.

Prior to measurement, further simulation results (not shown here) may describe the expected phasedifference as $\Delta \Phi(\varphi, d)$ diagrams, the expected visibility as $V(\varphi, d)$ diagrams or the Gain (shown below), etc. Examples of these auxiliary diagrams are given in [19 and 20].

\section{PDA for small particles}

As can be seen from the more general Fig. 3, two intrinsic problems arise for the PDA technique with respect to small particles with diameters comparable to $\lambda$ and below this value: a) there is a steady decrease of the scattered light intensity $I(d)$ with decreasing diameters; thus for a fixed detector sensitivity and with incident power restricted to a reasonable level, this fact limits the applicability of PDA as well as of any other optical sizing technique; and b) the phase-difference also decreases rapidly with decreasing particle diameters. Though $\Delta \Phi(d)$ can be shifted to higher values by increasing the elevation angles $\psi$ of the two detectors, a minimum $d$-value obtained from the final minimum resolvable phase difference nevertheless ultimately results. Actual minimum values depend on set-up parameters and detector sensitivity. As can be concluded from Fig. 3, conductive (metallic) particles yield higher phasedifferences than transparent ones in the submicron range, thus diminishing the above-mentioned difficulties significantly [21].
Two types of oscillations in the $\Delta \Phi(d)$ curves (Fig. 3) can be a severe hindrance to a direct conversion of a measured phase-difference to a sphere diameter. For transparent particles, interference between refracted and reflected scattering components causes these oscillations for $d<10 \mu \mathrm{m}$. The oscillations can be reduced to a minimum mainly by Brewster reflection suppression, via smoothing by means of large detector apertures or asymmetric set-up arrangements [22]. These "high-frequency" oscillations cause an increasing source of uncertainty in the data evaluation process.

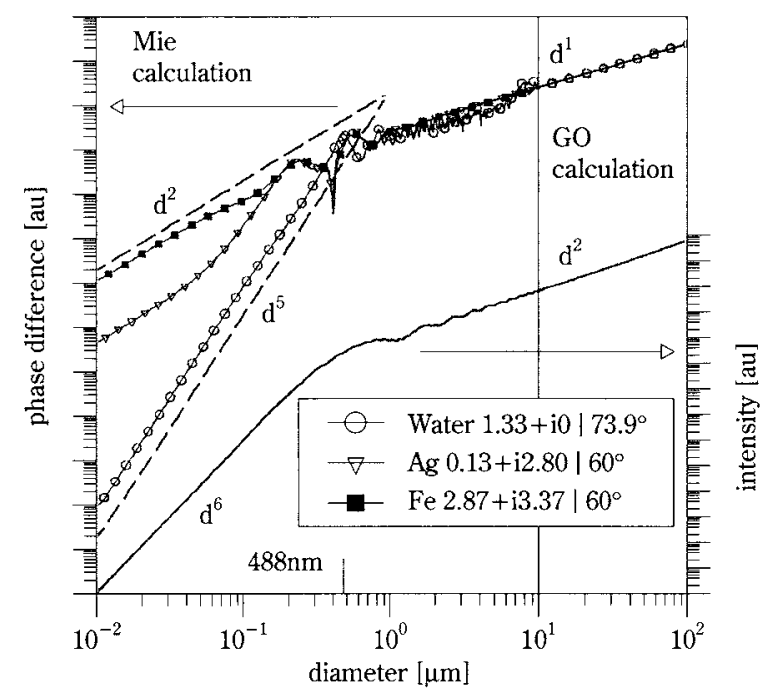

Fig. $3 \Delta \Phi$ (left axis) and scattered intensity $I$ (right axis) versus particle diameter $d$ for $\lambda=0.488 \mu \mathrm{m}$, various materials and off-axis angles $\varphi$. Mie calculations have been performed for diameters $d<10 \mu \mathrm{m}$ for water, $\mathrm{Ag}$ and Fe. For $d>10$ $\mu \mathrm{m}$, the geometrical optics results have been used

The other type of ambiguity can be observed from the small-particle limit for both transparent and absorbing particles. Here, rather "low-frequency" oscillations are obvious in the transition range between the exponential power law behaviour and the linear $\Delta \Phi(d)$ relation. This type of ambiguity can be reconstructed experimentally in detail [23]. Both types have to be considered during conversion of phase-difference to diameter.

Small-particle light scattering is associated with small scattering cross-sections, small phase-differences, and ambiguity in the form of oscillatory $\Delta \Phi(d)$ relations. This can be seen from Fig. 3, where $|\Delta \Phi|$ (holding true for both reflected and refracted light) and the scattered intensities $I$ are depicted versus $d$ 
Pfor a large diameter range in a log-log diagram [24]. Mie calculations have been performed for diameters $d<10 \mu \mathrm{m}$ for water (transparent), $\mathrm{Ag}$, and $\mathrm{Fe}$ (both with imaginary parts of the refractive index $m$ ). For $d>10 \mu \mathrm{m}$, the geometrical optics results (GO) have been used. Well-known limiting behaviour is obvious as well as $M i \ell$-specific resonances at $d \approx \lambda(0.488 \mu \mathrm{m}$, i.e. the laser wavelength).

\section{Non-homogeneous droplets}

\subsection{Superfine inclusions}

Although water atomization may play an important role for, e.g. cooling processes or model experiments, there is also a great demand for spray analysis of real process fluids such as paints, emulsions or suspensions, which have to be atomized in order to yield sufficiently smooth or colourful surfaces or to produce dry and small samples of good consistency. These types of process fluids contain inhomogeneities of varying materials and shapes and of mostly typical size ranges due to the previous process steps. As mentioned above, difficulties arise if the droplets to be measured contain such optical inhomogeneities, because the linear phase-difference/particle diameter relation may be limited or is no longer valid. The size of these inclusions in the bulk fluid differs greatly, but can be classified into superfine particles - of the order of, or smaller than, the wavelength of the laser light $\lambda$ - and large particles - reaching the range of the droplet diameters. Their refractive index is different from that of the bulk fluid of the droplet, thus influencing the effective refractive index of the composite medium. Since the refractive index of the inhomogeneities usually contains an absorption term (factor $k>0$ of the imaginary part), this consequently means that the composite medium behaves like a more or less absorbing fluid. As long as these emulsion or suspension droplets are spherical in shape without any bulge in the surface (which might be a result from higher concentrations), PDA can principally be used for spray or droplet analysis [25 to 27].

When using a PDA system to characterize inhomogeneous droplets, the size distributions that are measured are artificially broadened compared with those size distributions of droplets which contain only the pure bulk liquid (without any inhomogeneities). This artificial broadening effect can in most cases be reduced by the proper choice of the laser wavelength [28 and 11]. If not, it was shown that the application of highly sophisticated mathematical algorithms enables the PDA user to reconstruct the real size distribution from the measured one by using the information about the measured droplet size distribution of monosized droplets produced from the pure bulk liquid by a droplet generator, as shown in Fig. 4 [29 to 31]. Using the Dual-Burst-PDA, it could be shown that it was possible to extract additional information about the concentration of the inhomogeneities from inside the droplet.

Due to the disturbing effect of the dispersed phase inside the droplet on the light scattering, a linear correlation between the phase-difference of the refracted light and the diameter of a single droplet does not, in most cases, exist any longer. Such a correlation only exists for the mean value of all the measured droplets. This effect is obvious when measuring high numbers of droplets of identical diameter. As can be seen from Fig. 4, the PDA measurements of 10,000 monosized droplets of either pure bulk liquid or bulk liquid con-
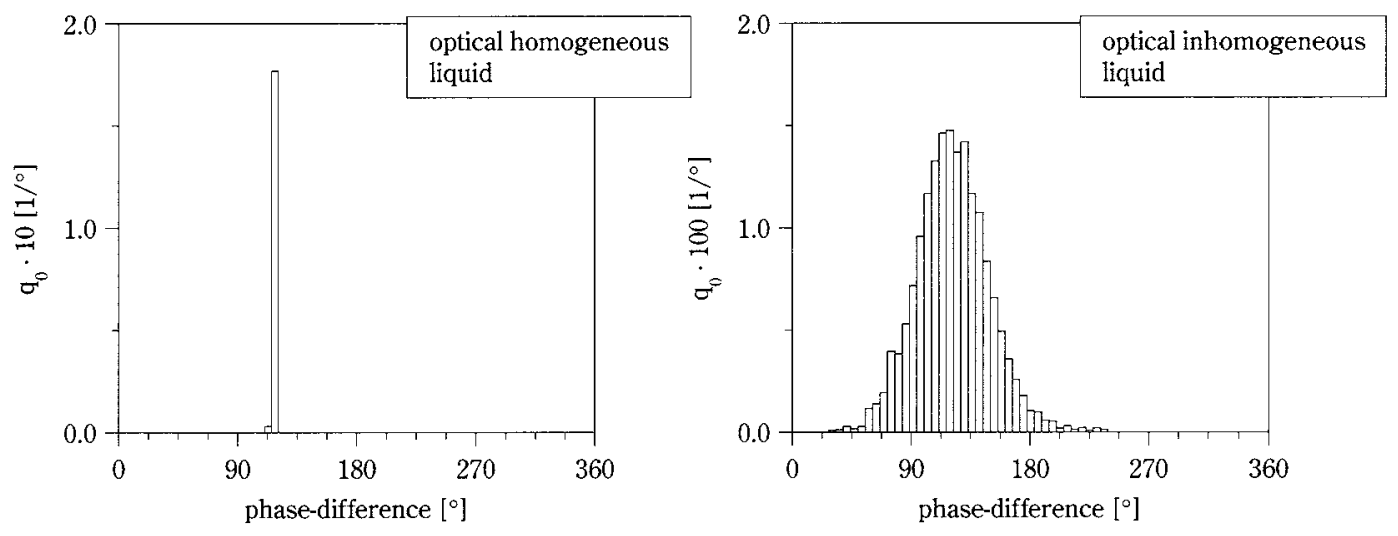

Fig. 4 PDA measurements from monosized optically homogeneous droplets and from droplets of the same bulk liquid - but containing fine inhomogeneities 
taining fine inhomogeneities result in quite different distributions. In the case of optically homogeneous droplets, PDA detects nearly the same phase-difference for every droplet. In the case of optically inhomogeneous droplets, the measured phase-difference distribution is broadened into a Gaussian function. Using - as usual - only a linear $\Delta \Phi(d)$ correlation to compute the droplet diameter from these phase differences, nothing other than a Gaussian distribution is achieved, which is a false interpretation. On the other hand, the simultaneously measured droplet velocity is almost independent of the optical character of the droplets

The standard deviation of such a Gaussian distribution depends on the diameter of the dispersed inhomogeneities, on their concentration, and on the parameters of the PDA set-up. Regarding the phasedifferences, their spreading can be influenced by the proper choice of a smaller beam-crossing angle $\phi$ and by off-axis angles $\varphi$, describing the domain of reflection (as will be discussed below). The standard deviation is not affected by the elevation angles of the detectors.

The Mie theory and other light scattering theories have been expanded in order to simulate the scattering behaviour, but their range of application is still limited either to a few larger particles in the droplet or to droplets with significantly smaller inclusions compared with $\lambda$. Recently, by using an independent scattering approximation [32], it was possible to simulate the broadening effect for a latex concentration $(\mathrm{d}=2 \mu \mathrm{m})$ of 2 vol.\%. These simulation results agree perfectly with measurement results of the broadening obtained from monosized droplets of a latex suspension produced by a piezoelectric droplet generator.

In spite of this theoretical progress, in most technical applications, inhomogeneities cover a large section within the size range of the laser wavelength. While for monosized droplets the problem seems to be clear and quite easy to discuss, the broadening effect needs further interpretation of the PDA results in the case of polysized droplet sprays of optically inhomogeneous (process) liquids, which normally occur in industrial atomization processes.

Such sprays of solutions, e.g. of coffee and condensed dairy milk (inhomogeneous), in comparison with those of pure water and ink (homogeneous), have been analysed under various - but identical atomization conditions, using laser wavelengths of between 0.488 and $0.830 \mu \mathrm{m}[25,26$ and 11]. The question was whether the inhomogeneous liquids can be treated as homogeneous ones by using an effec- tive complex refractive index of the composite medium instead of the refractive index of the bulk liquid (mostly water or an organic phase). The answer is that this can be done not only for spherical inclusions (milk), but also for non-spherical ones (coffee), as long as the inclusions are comparable to or smaller than the wavelength of the laser light. But the procedure to estimate the complex relative refractive index of an optically absorbent multicomponent liquid is complicated [11].

These results can be explained by Fig. 5a), where the integrated light intensities per diffracted, refracted and reflected light components are given as Gain versus off-axis angle $\varphi$. An increasing value of the absorbent factor in the refractive index $k$ (from 0.001 to 0.010 ) diminishes the importance of the refracted light, whereas the curves of the diffracted $\left(\varphi<36^{\circ}\right)$ and reflected light $\left(0^{\circ}<\varphi<180^{\circ}\right)$ remain constant.

If one is forced to detect the scattered light of the droplet (multicomponent liquid) from a forward direction (i.e. the refracted light), this becomes more and more problematic caused, e.g. by an increase of concentration of the inhomogeneities.

Here, one gets another degree of freedom by changing the type of laser, i.e. by applying a laser with a different wavelength $\lambda$, thus one can change the Mie parameter $\alpha=\pi d / \lambda$, i.e. the ratio between particle circumference and wavelength. This can be explained by Fig. $5 \mathbf{b}$ where an increase of the particle diameter $d$ causes nearly the same effect (as demonstrated in Fig. 5a by the augmentation of the $k$ value). The particle size, given as a Mie parameter $\alpha$ varies from 25 to 250 . The importance of the refracted light decreases with increasing particle diameter, whereas the curves of the diffracted $\left(\varphi<36^{\circ}\right)$ and reflected light $\left(10^{\circ}<\varphi<180^{\circ}\right)$ remain constant. Vice versa this means, if the laser wavelength can be changed to higher values of $\lambda$, one can remove the problem of absorption of light in the range of visible wavelengths [29 and 33].

This depends - as demonstrated above - on the liquid's absorption properties and allows the linear $d(\Delta \Phi)$ correlation (Equation (1)) to be used. Extrapolating these results - in the case of optically absorbent inhomogeneous liquids - the use of a larger wavelength might be universally advantageous because it reduces scattering by these inhomogeneities inside the droplets. But, as has been shown in [29 and 33], changing to larger wavelengths does not always lead to more realistic results.

On the contrary, the proper selection of the laser 
Pwavefength once again has to be based on Mie calculations of the extinction efficiency. Larger wavelengths are the better choice only for low Mie parameters $(\alpha \leq 5)$, dependent on the refractive index. For larger values of the Mie parameter, the relation between the extinction efficiency and the wavelength is ambiguous. In other words, as long as the light intensity is sufficient for PDA analysis, droplets of optically absorbent multicomponent liquids should be analysed under off-axis angles where the reflected
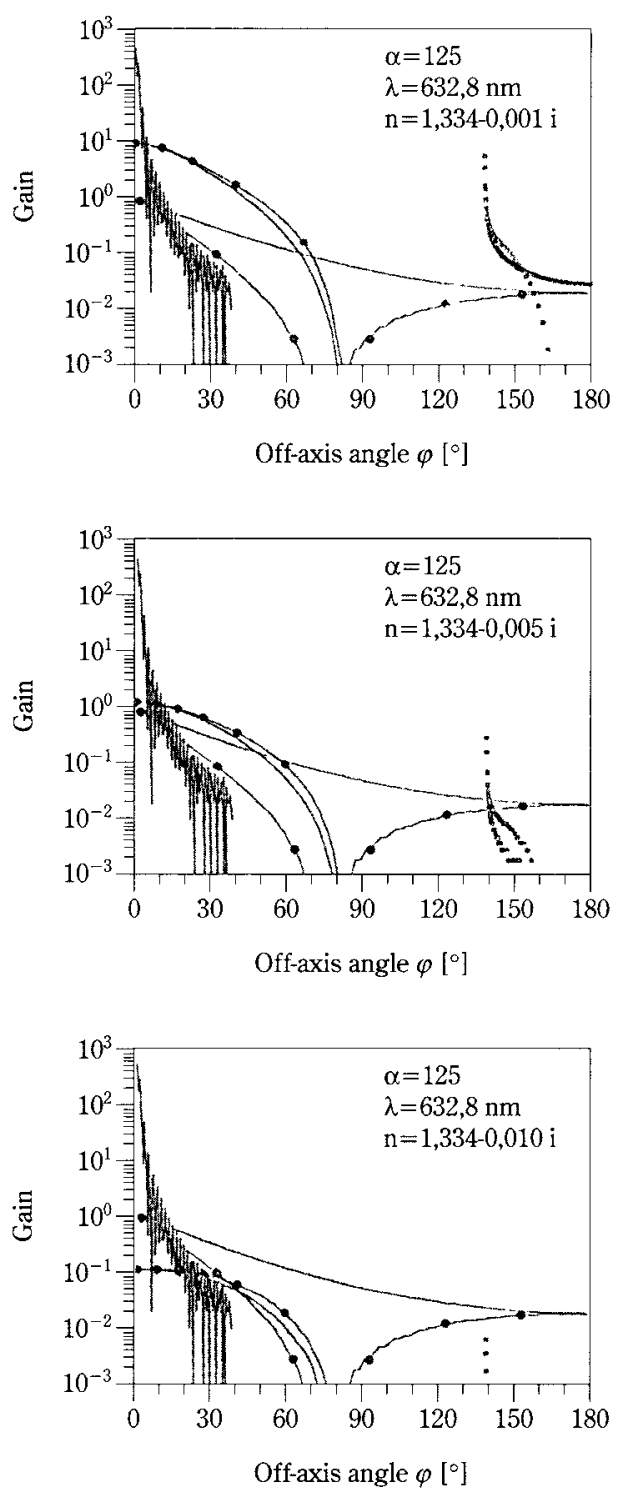

a) light component is dominant.

The experiments with optically absorbent multicomponent media [29 and 33] disclosed the tremendous potential for misinterpretation of PDA results and false droplet size distributions. In addition to milk and coffee and concentrated solutions of latex-water suspensions of definite monosized particles of 0.45 , 0.72 and $3.50 \mu \mathrm{m}$, the laser wavelengths 0.488 and $0.830 \mu \mathrm{m}$, as well as 0.632 and $1.312 \mu \mathrm{m}$ [33 and 34], were used. For each of these liquids, the results made
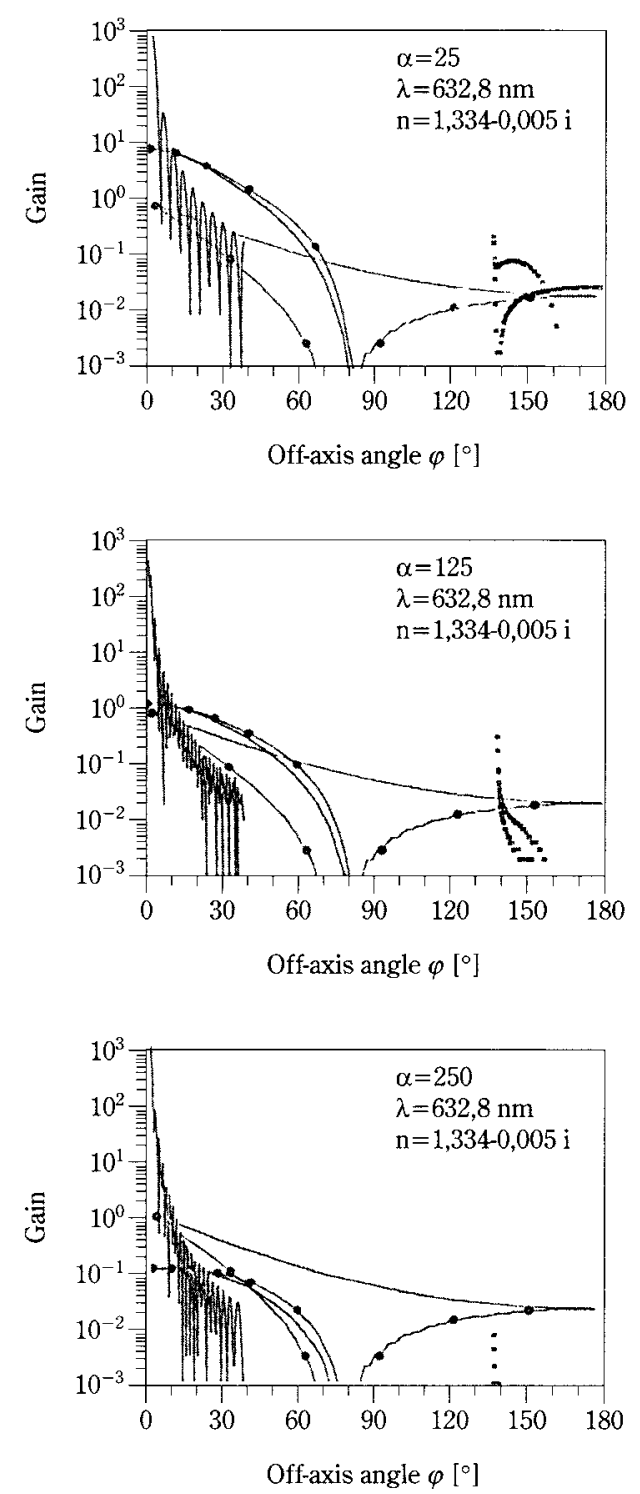

b)

Fig. 5 Gain versus off-axis angle for different Mie parameters $\alpha$ and absorption factors $k$ 
it ecessary to apply mathematical inversion algorithms to finally bring the widely spread data to a realistic droplet size distribution of the measured spray.

\subsection{Inclusions in the diameter range of the droplet}

Research work in meteorology, medicine, and pharmacy also deal with small droplets containing larger inclusions. PDA applications are, for instance, focused on generation processes of microcapsules which allow ultrasonic diagnostics of the blood circulation, or on capsules that include pharmaceuticals or coloured ink, as well as on coated spheres, e.g. watercoated carbon core particles. Such "droplets" with spherical inclusions that match the range of the droplet diameter cannot be treated as homogeneous ones with an effective complex refractive index (of the composite medium), because these inclusions reveal their own individuality. From the optical point of view, the material combinations of shell and core can be classified as a transparent or absorbent continuous phase including a transparent or absorbent dispersed phase. Research work has been carried out with several of these combinations, defined by their refractive index, respectively [35 to 39]. Using the generalized Lorenz-Mie theory, it could be shown by simulating the response of a PDA device that not only the core, but also the outer diameter of the particle could be detected as well as the refractive index profile of a multilayered particle [35].

Replacing the two detectors of the PDA system by a CCD line-scan sensor with, e.g. 256 pixels, allows the detection of 256 signals instead of two, thus capturing the scattered light with high spatial resolution. The phase-difference can be obtained 128 times, using two symmetrical (in relation to $\psi=0^{\circ}$ ) pixels respectively as a pair. The CCD line-scan sensor can also be arranged with all its pixels in the $\left(\psi=0^{\circ}\right)$-position, i.e. in the horizontal position instead of the vertical one (Fig. 6) [36]. Generally, the evaluation of the resulting signals can be performed in two different ways. The evaluation is either based on the entire signal or on selected parts of the burst, for example the lines of highest intensity in the temporal and spatial dimensions. Using the first alternative, detailed information can be obtained from the scattering process and thus from the particle passing the interference volume. The disadvantage is the high computational time required. The other option, evaluating just one line from the burst, is very fast but rather prone to error.

As outlined in a detailed previous description, using simulation results as well as results of various experi- ments [39], it is possible to analyse the diameter as well of the core as of the enclosing droplet, thus giving information on the shell thickness, preferring the horizontal arrangement of the CCD line-scan sensor, whereas both arrangements have the potential of exact size determination of, for instance, solid spheres with inhomogeneous composition (glass beads with cracks) or surface defects, whereas the measurable particle velocity for both arrangements is limited at present to approximately $10 \mathrm{~ms}^{-1}$, because of the low scan rate of the CCD camera available.

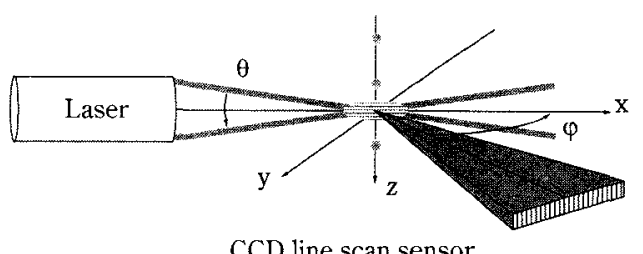

CCD line scan sensor

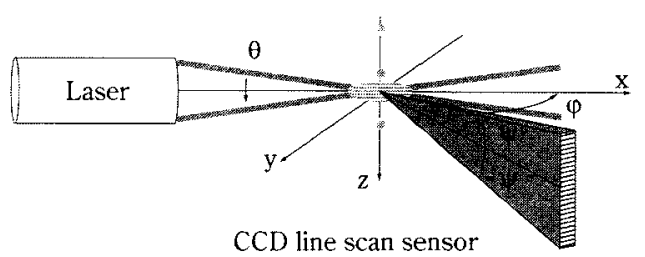

Fig. 6 PDA arrangements with a horizontal or vertical CCD linescan sensor [39]

\subsection{Droplets with radial gradients of the refractive index}

Droplets of two or more completely miscible liquids, which are influenced by heat and mass transfer processes (liquid/liquid-extraction, spray combustion, evaporation), show a variation of their integral concentration (average value) with time, causing also a variation of their effective (average) refractive index with time. If their Biot number ${ }^{1}, B i$, exceeds values of about 1 , radial concentration gradients (depending on the material properties of their components) also emerge that cause radial gradients of the effective refractive index of the composition. The change of the average value with time, as well as the variation of the

${ }^{1} B i=h d / K_{t p}$ for heat transfer with $h=$ heat transfer coefficient and $K_{t p}=$ thermal conductivity of dispersed phase $B i=\beta d / D_{p}$ for mass transfer with $\beta=$ mass transfer coefficient and $D_{p}=$ molecular diffusity of dispersed phase 
Pgradients with time, depends on the intensity of the process and on the specification of the components. Both effects can be measured simultaneously, together with particle size and velocity [35; 40 and 41], and can be used for in-line process control. Since a direct correlation exists between the average refractive index and the droplet temperature, these PDA measurements are principally also suitable for inflight temperature measurements. But improved precision can only be reached by using rainbow interferometry [ 42 to 44$]$.

\section{Deformed spheres}

\subsection{Spheroidal droplets}

Deformation of spherical droplets takes place during droplet generation near a nozzle or within a gaseous atmosphere during accelerated and decelerated droplet motion, whereas even very small forces during droplet motion or during mass transfer may create deviations from the spherical shape within a liquid environment. In the case of axisymmetrical deformation one speaks of spheroids. Since light scattering is influenced mainly by the curvature of the particle surface (liquid/gas or liquid/liquid interface), these deformations create various light patterns from the same particle that result in deformed PDA bursts, leading to ambiguous signal interpretation. In addition, droplet oscillations (causing prolate or oblate shapes) or droplet deceleration and acceleration within the measuring volume give rise to similar burst deformations.

Using Fast-Fourier-Transform (FFT)-based signal processing allows an improvement of the signal validation, time-resolved frequency, and phase-difference analysis from the two bursts, and thereby the determination of the burst envelope and phase-difference changes, which results in additional information about the non-spherical droplet character while passing the measuring volume [ 45 to 47 and 44]. In Fig. 7 , simulated results show the variation of the phasedifference $\Delta \Phi$ within the bursts of a prolate or oblate droplet with diameters $d_{v}$ and $d_{h}$. In the case of $d_{v}=d_{h}$ with $E=d_{h} / d_{v}=1$, the phase-difference $\Delta \Phi$ of an equivolumetric spherical droplet of a diameter of $2 \mathrm{~mm}$ is $254^{\circ}$.

A comparison between standard PDA and a planar detector arrangement [48], where the two detectors are placed at (two) elevation angles in the plane of the transmitting beams, demonstrated that non-sphericity of droplets leads to significant changes in the phasedifference, much more so for prolate than for oblate

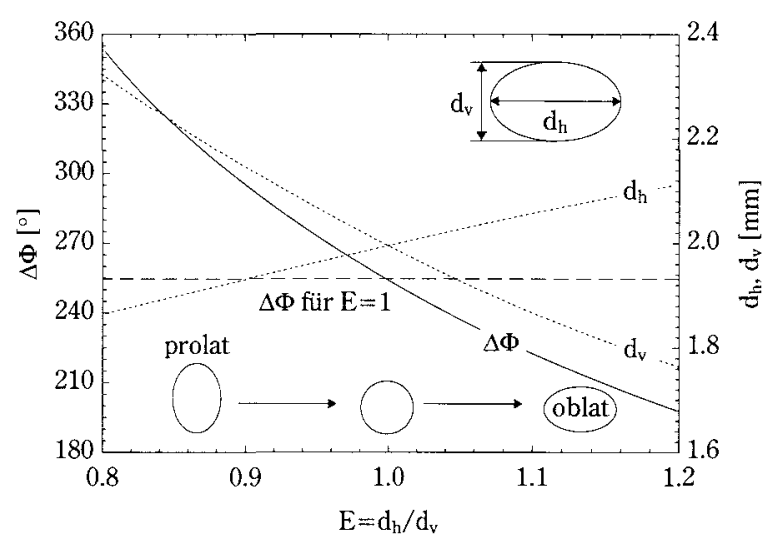

Fig. 7 Computed phase-difference $\Delta \Phi$ (and therefrom diameters $d_{v}$ and $d_{h}$ ) of deformed droplets versus quotient $E$ of horizontal to vertical droplet diameter after [44]

droplets, and stronger for the standard detector arrangement than for the planar detector arrangement. Increasing the investment, the combination of both standard and planar detector arrangements finally leads to more accurate information about the deformation [48].

\subsection{Spheroidal fine particles}

Theoretical [49] as well as theoretical and experimental work [50] has been directed at recognizing the potential and the limitations of PDA measurements of fine spheroids. Monodispersed polystyrene latex particles (equivolumetric spheres of diameters of 3.0 and $3.5 \mu \mathrm{m}$ ) were stretched to a predetermined extent in order to convert the spherical particles to spheroids with semi-axes $a=(d / 2) \varepsilon^{2 / 3}$ and $b=(d / 2)$ $\varepsilon^{1 / 3}$ [49]. These particles were measured as single particles by means of an electrodynamic trap [23] as well as suspended ones in double-distilled water (as flowing suspensions). As can be seen from Fig. 8, the electron micrograph shows well-defined monodispersed (polystyrene) spheroids, and the frequency distributions of phase-differences show a good agreement between experiment and simulation.

While liquid droplets (or polystyrene particles) due to their surface tension - are in general spherical or at least spheroidal in shape, solid (metal) particles show more or less (local) deviations from sphericity. Their surface exhibits structures resulting from the solidification and/or crystallization process. The real particle properties can neither be quantified in detail - in addition there are differences from one particle to another - nor can they be accounted for exactly in the light scattering computations. There- 

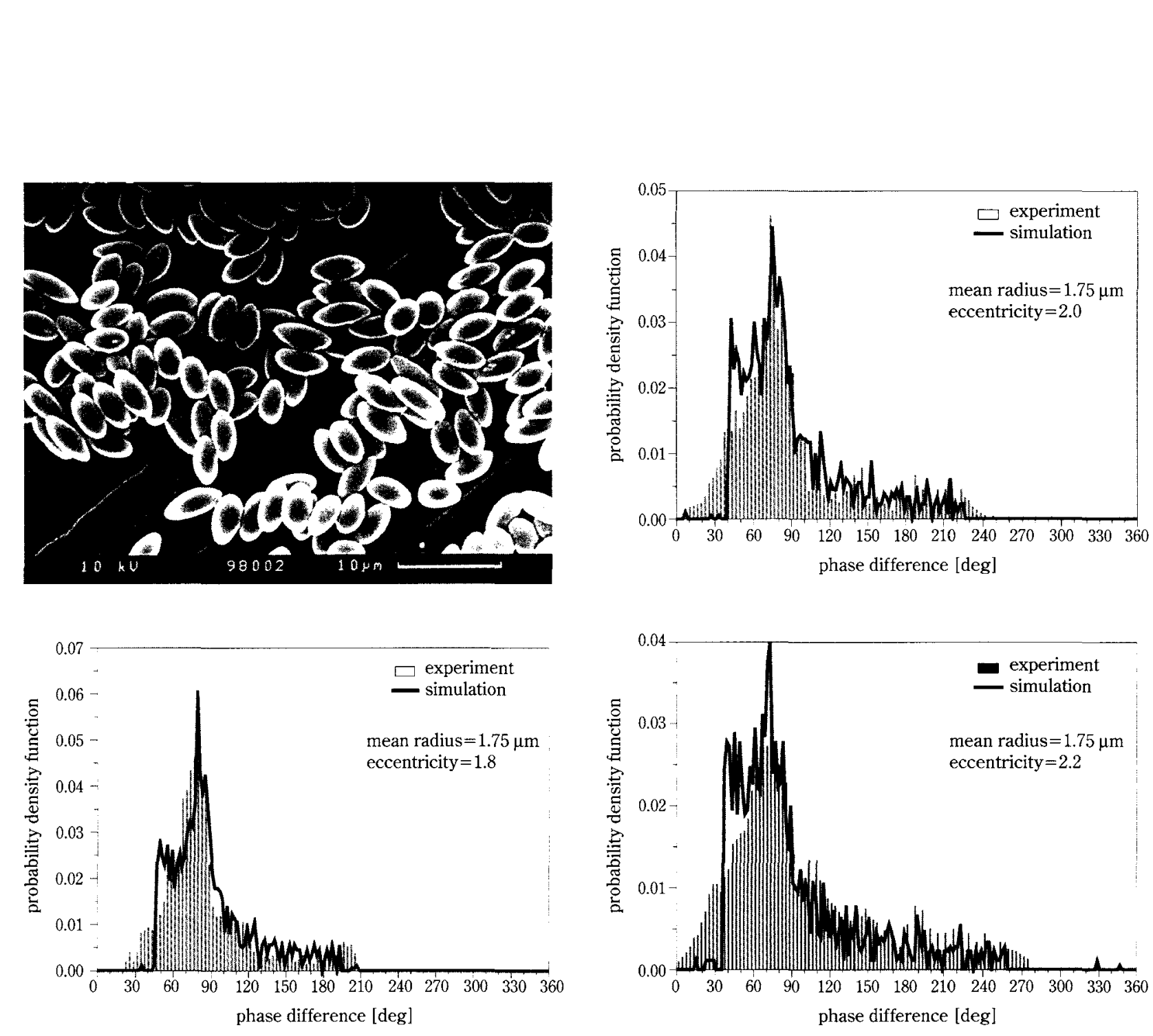

Fig. 8 Electron micrograph of monodispersed polystyrene spheroids and frequency distributions of phase-differences of these particles (measured and simulated results) for $d=3.5 \mu \mathrm{m}$ and $\varepsilon=1.8,2.0$ and 2.2 in accordance with [49]

exactly in the light scattering computations. Therefore, an apparent decrease of precision during the PDA measurements of fine solid particles has to be tolerated which results in a broadening of the measured phase-difference distributions, thus leading to an apparent inaccuracy of diameter detection and corresponding particle size distribution.

\subsection{Surface roughness and deviations from sphericity of solid particles}

In order to analyse the influences of surface roughness and deviations from sphericity and to quantify the problems of apparent inaccuracy of diameter detection, single fine solid particles were measured several times using an electrodynamical particle trap [23]. After capturing the individual particles from the trap, the resultant mean particle characteristics were compared by electron microscopical observation [23, 51 and 52].
Several measurements on micron and submicron monospheres - both transparent and conducting on monodisperse aerosols, and on fine metal particles verify the theoretical and numerical results. Fig. 9 shows number density distributions $q_{d}(\Delta \Phi)$ (performed by $10^{4}$ single diameter measurements on each particle) from four solid spherical particles: two steel particles (St2 and St4), a tin particle (Sn5), and a copper particle (Cu1). Although the same particles were analysed in each case, the results show typical number density distributions which can be regarded as an orientation-averaged PDA result in any case. Especially the width of the distributions gives a measure for the deviations from sphericity. In addition, the surface structures have a further broadening effect on the width of these distributions. Thus, the apparent decrease of precision during PDA measurements of solid particles has a complementary result: together with the information of the mean particle diameter, 

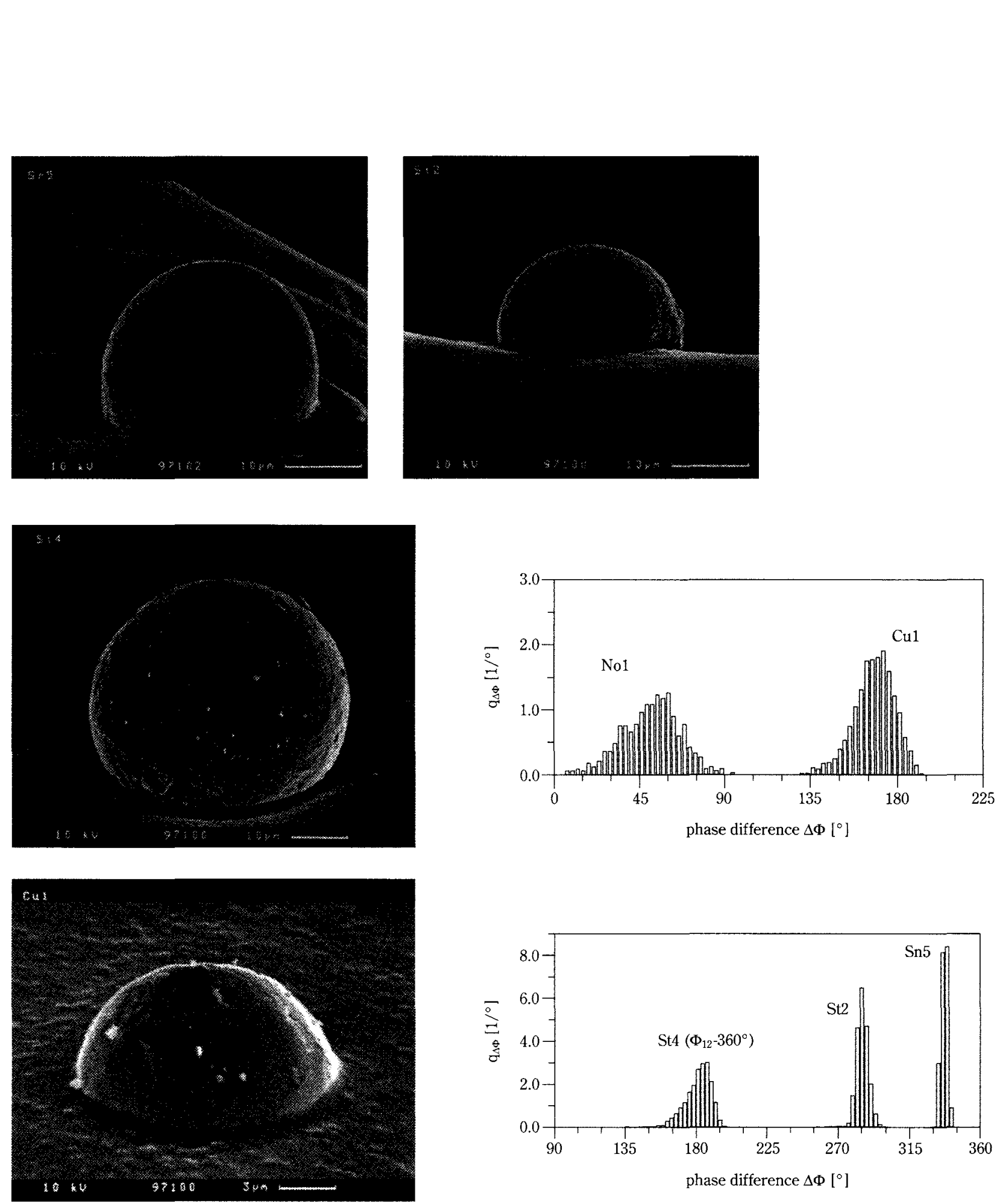

Fig. 9 PDA-measured number size distributions of three metallic particles as per [23]

one can obtain information about its sphericity and surface structures.

The particles were submerged in a conducting glue to some degree before electron microscopical photographs were taken. From these photographs, the mean particle diameter $d_{R E M}$ was extracted and compared with the PDA results $d_{P D A}$. The agreement is good in all cases, though the width of the PDA num- ber density distributions varies from particle to particle. After correlating the width of each measured diameter distribution with the surface roughness, one could observe from the electron microscopical photographs (same figure) some surface structures as well as some impurities sticking on the surface. This was the explanation for the broadening effect.

Since the tin particle ( $\mathrm{Sn} 5)$ exhibits the smoothest 
$P$ sunface, the width of its diameter distribution is small $\left[d_{R E M}=(28.2 \pm 0.5) \mu \mathrm{m} ; \Delta \Phi=335^{\circ} ; d_{P D A}=(27.8 \pm 1.6)\right.$ $\mu \mathrm{m}]$. The surface roughness is slightly higher for the steel particle St2 $\left[d_{R E M}=(23.6 \pm 0.5) \mu \mathrm{m} ; \Delta \Phi=291^{\circ}\right.$; $\left.d_{P D A}=(24.2 \pm 1.3) \mu \mathrm{m}\right]$ and again for the steel particle St4 $\left[d_{R E M}=(45.5 \pm 0.7) \mu \mathrm{m} ; \Delta \Phi=177^{\circ} ; d_{P D A}=(44.6 \pm\right.$ 2.5) $\mu \mathrm{m}]$. The worst measurement result was from the copper particle $\left[d_{R E M}=(14.4 \pm 0.2) \mu \mathrm{m} ; \Delta \Phi=167^{\circ}\right.$; $\left.d_{P D A}=(13.9 \pm 0.8) \mu \mathrm{m}\right]$ and from a Novamet particle No1 with substructures in its surface, not shown here $\left[d_{\text {REM }}=(5.52 \pm 0.19) \mu \mathrm{m} ; \Delta \Phi=71.1^{\circ} ; d_{P D A}=(5.9 \pm 0.3)\right.$ $\mu \mathrm{m}]$. Accordingly, the measured diameter distributions are broadened, which could be reconstructed by means of a ray theory model for spheres with random shapes and a computation of the far-distance electric fields and PDA phase-differences [51 and 52].

Because these results are in good agreement with the experimental data given above, one can conclude - generally speaking - that powders consisting of spherical particles can principally be measured by PDA, if it is sufficient to obtain information on the mean values of the particle diameter or about the average characteristics (quality) of the product.

\section{Agglomerates of solid spheres}

Since the formation of agglomerates in general gives rise to problematic size distributions of powders, and because substances such as solders, consisting of fine spherical metal powders, require particles free of agglomerates, there is a great demand for process control, e.g. of gas atomization of metal melts, where the metal powder production results in fine spherical particles. In principle, a tool for this process control, i.e. for the control of agglomeration or of agglomeration-free powder production, can be PDA (if further research work is performed). After solving the fundamentals of light scattering from a two-sphere system in the intersection field of the PDA (probe volume), mathematical tools, based on the approximate ray theory, were developed and the phasedifferences simulated for a real three-sphere system and compared with such an agglomerate [53]. The agreement between simulation and experiment is good as long as the spheres are almost the same size. Otherwise, a single peak, corresponding to the largest sphere, occurs in the frequency distribution, and the smaller particles will broaden the phase distribution.

\section{Dense sprays}

The denser the sprays, i.e. the higher the particle fluxes (or concentrations) and the stronger the extinction of the atomized medium, the lower will be the probability of measuring realistic mass flux rates within the spray by PDA.

Dense sprays can be defined either as two-phase flows with high particle (mass or volume) fluxes of the droplet phase (e.g. water), thus increasing the probability of coincidences of droplets, passing the PDA probe volume, or - from an optical point of view - as two- or multi-phase flows with an increased optical extinction (i.e. absorption and scattering) of the entire dispersed phase, due to the material properties of the atomized droplets and their increased scattering probability. The scattered light from the droplet(s) in the probe volume will be influenced by other droplets, simultaneously passing either the probe volume or the laser beams or the light paths to the detectors, not to mention that the signal-to-noise ratio (SNR) will become worse in any case. The increased optical absorption of the entire droplet phase, also due to the increased droplet concentration, depends on the material properties of the atomized medium, originating from a high value of the factor $k$ of the imaginary part in the refractive index, if the medium is homogeneous (e.g. metal melt droplets) or from inclusions of the droplets, when the effective refractive index results from a composite medium (described in Section 5.1). For instance, the atomized medium may be a process liquid like a suspension or an emulsion.

In general, the original size distribution of the droplets after atomization ranges over more than two decades, thus a low SNR may already cause an underrepresentation of the finest particles in particular. This tendency may be intensified by increased optical extinction and/or multiple scattering of high particle fluxes. Since in axisymmetrical sprays, the local particle flux changes with distance not only from the atomizer but also from the centre line, this causes longitudinal and radial gradients of density, of various local SNR, and of multiple scattering probability. In addition, some of the above-mentioned problems may also diminish the signal quality of the droplets: inclusions, pulsations together with deformations, or agglomerates and surface structures after solidification of melt droplets. Thus the assumption that PDA measurements from single particles may provide absolute measures has to be abandoned, because particle collectives such as sprays or powders in most cases show a strong tendency to have their finer particles under-represented and their larger particles over-represented (due to agglomerates). 
In or der to eliminate these types of errors, one can use additional instrumentation such as patternators or diffraction techniques or laser light sheet arrangements. Also, the choice of different lasers with appropiate wavelengths - as described in Section 5.1 may solve some of these problems. An entirely different strategy is the use of post-processing programs, as have been developed, e.g. for dense metal melt sprays [54 and 55] and sprays of optical absorbing process fluids [33], or to combine special PDA arrangements with a post-processing program, in order to eliminate coincidence [56]. In any case, post-processing needs a fast signal acquisition for definite time periods without using any trigger criteria. Hence a kind of signal band is recorded, including bursts as well as noise. This signal band is stored on a hard disk (without any data evaluation) for subsequent signal analysis. It is intended to coordinate the various ways of data evaluation (described in the foregoing sections) and to implement them in a common evaluation scheme and upgraded post-processing program.

\section{Conclusions}

An extended application of standard phase-Doppler anemometry (PDA) to deformed or opaque (absorbing) particles, to metal melt droplets, or to droplets which contain inclusions - so called optical inhomogeneities - has been discussed. Since problems may also occur due to the surface roughness or to agglomeration of solid particles, it is in any case helpful to use light scattering simulations based on geometrical optics (GO) and the Mie theory for the design and arrangement, and finally for optimisation, of the optical set-up prior to measurements.

Experiments have been described with spherical particles of different materials: polystyrene, coffee suspensions, and milk emulsions, molten and solidified steel and tin. Since solid particles show more or less deviations from sphericity, the width of PDAmeasured diameter distributions gives a measure for these deviations. Since the surfaces of solid particles also exhibit structures resulting from the solidification and/or crystallization process or even contain impurities, these too have a further broadening effect on the width of these distributions. Hence, the apparent decrease of precision of PDA measurements from fine solid particles had to be cleared up, ending in a complementary result: together with the information of the mean particle diameter, one also obtains information about its sphericity and surface structures or - generally speaking - of the product quality.
All these strategies have to be tested, and can be coordinated, in order to implement the various data evaluations in a common post-processing scheme.

\section{References}

1) E.D. Hirleman: Part. Part. Syst. Charac. 13 (1996) 59-67;

2) K. Bauckhage; H.-H. Flögel; U. Fritsching; R. Hiller: Part. Part. Syst. Charac. 5 (1988) part 1: pp. 16-22; part 2: pp. 66-71;

3) K. Bauckhage: Chem.-Ing.-Tech. 68 (1996) Nr. 3, S. 253266 ;

4) C. Tropea; T-H. Xu; F. Onofri; G. Gre’han. P. Haugen; M. Stiegelmeier: Part. Part. Syst. Charac. 13 (1996) 165170 ;

5) P. Lehmann; E.H. Schombacher; Th. Wriedt: Part. Part Syst. Charac. 13 (1996) 27-33;

6) G. Gre'han; G. Gouesbet; A. Naqwi; F. Durst: Part. Part. Syst. Charac. 11 (1994) 133-144;

7) K. Bauckhage: Chem.-Ing.-Tech. 65 (1993) Nr. 10, S 1200-1205;

8) H.-H. Flögel: Dissertation Universität Bremen (1987), Fortschr.-Ber. VDI Z. Reihe 3, Nr. 140, VDI Verlag, Düsseldorf, 1987;

9) SCATAP-Programm: WWW http://diogenes.iwt.unibremen. de/vt/laser/laser/htm1;

10) Z. Jiang: Dissertation Universität Bremen (1994);

11) U. Manasse: Dissertation Universität Bremen (1993), Fortschr.-Ber. VDI Z. Reihe 8, Nr. 353, VDI Verlag, Düsseldorf, 1993;

12) M. Willmann; A. Glahn; S. Wittig: Part. Part. Syst. Charac. 14 (1997) 122-128;

13) K. Bauckhage; F. Schöne; D. Dopheide: Int. Conf. on Laser Anemometry, Advances and Applications, Swansea, Wales 1989;

14) J. Tillwick: Dissertation Universität Bremen (1999);

15) J. Tillwick; V. Uhlenwinkel; K. Bauckhage: Int. J. Heat and Fluid Flow 20 (1999) 530-537;

16) P. Haugen; E.J. Hayes: 3rd Int. Congr. on Opt. Particle Sizing; Yokohama 1993;

17) P. Haugen; E.J. Hayes; H.-H. von Benzon: Part. Part. Syst. Charac. 11 (1994) 63-72;

18) H. Schombacher; Th. Wriedt; K. Bauckhage: PARTEC $1995-4^{\text {th }}$ Int. Congr. on Opt. Particle Sizing; Nürnberg 1995 ;

19) Z. Jiang: Applied Optics 36, 6 (1997) 1367-1375

20) P. Buchhave; H.-H. von Benzon: Part. Part. Syst. Charac. 13 (1996) 68-78

21) G. Göbel; Th. Wriedt; K. Bauckhage; Journal of Aerosol Science, Vol. 29, No. 9, pp 1063-1073; 1998

22) H.-H. von Benzon; P. Buchhave: Part. Part. Syst. Charac. 11 (1994) 55-62;

23) G. Göbel: Dissertation, Universität Bremen 1998

24) G. Göbel; Th. Wriedt; K. Bauckhage: Rev. Sci. Instrum. 68 (8) August 1997; pp 3046-3052

25) U. Manasse; Th. Wriedt; K. Bauckhage: Part. Part. Syst. Charac. 9 (1992) 176-185

26) U. Manasse; Th. Wriedt; K. Bauckhage: Meas. Sci. 
N'echnol. 4 (1993) 369-377

27) F. Onofri; L. Bergougnoux; J.-L. Firpo; J. MisguichRipault: Applied Optics; 20. July (1999), Vol 38, No 21, 4681-4690

28) U. Manasse; Th. Wriedt; K. Bauckhage: Part. Part. Syst. Charac. 11 (1994) 84-90

29) M. Mitschke; Th. Wriedt; K. Bauckhage: Meas. Sci. Technol. 9 (1998) 197-209

30) Th. Wriedt; M. Mitschke; P. Lehmann: SPIE, Proc. Optical Techniques, Thermal and Combustion Flow; San Diego, July 10-13; Vol. 2546; (1995)

31) Th. Wriedt; M. Mitschke; U. Fritsching; K. Bauckhage: $2^{\text {nd }}$ Int. Conf. on Multiphase Flows; Kyoto, Japan; April 3-7; 1995

32) A. Doicu; M. Mitschke; Th. Wriedt; K. Bauckhage: Part. Part. Syst. Charac. Submitted.

33) M. Mitschke: Dissertation Universität Bremen (2000), Fortschr-Ber. VDI $Z$. Reihe 8, Nr. 852, VDI Verlag, Düsseldorf, 2000

34) O. Köser; Th. Wriedt: Applied Optics 35, 15 (1996) 2537 2543

35) F. Onofri, D. Blondel; G. Gre'han; G. Gouesbet: Part Part. Syst. Charac. 13 (1996) 104-111

36) J. Rheims; H. Dahl; Th. Wriedt; K. Bauckhage: PARTEC $1995-4^{\text {th }}$ Int. Congr. on Opt. Particle Sizing, Nürnberg 1995

37) J. Rheims; Th. Wriedt; K. Bauckhage: Meas. Sci. Technol. 10 (1999) 68-75

38) J. Rheims; Th. Wriedt; K. Bauckhage: Part. Part. Syst. Charac. 15 (1998) 219-224

39) J. Rheims: Dissertation Universität Bremen (1998), Fortschr.-Ber. VDI Z. Reihe 8, Nr. 710, VDI Verlag, Düsseldorf, 1998

40) S. Sankar; K.M. Ibrahim; D.H. Buermann; W.D.
Bachalo: 3rd Int. Congr. on Opt. Particle Sizing; Yokohama 1993

41) W.D. Bachalo: Part. Part. Syst. Charac. 11 (1994) 73-83

42) L. Kai; P. Massoli; A. D'Alassio: Part. Part. Syst. Charac. 11 (1994) 385-390

43) K. Anders; N. Roth; A. Frohn: Part. Part. Syst. Charac. 13 (1996) 125-129

44) H. Schombacher: Dissertation, Universität Bremen 1997; Fortschr.-Ber. VDI Z. Reihe 8, Nr. 648, VDI Verlag, Düsseldorf, 1997

45) P. Lehmann; Th. Wriedt; A. Schöne: SPIE, Proc. Optical Techniques, Thermal and Combustion Flow; San Diego, July 10-13; Vol. 2546; 246-257 (1995)

46) A. Doicu; Th. Wriedt; K. Bauckhage: Part. Part. Syst. Charac. 14 (1997) 3-11

47) P. Lehmann; H. Schombacher; Th. Wriedt: Part. Part. Syst. Charac. 13 (1996) 27-33

48) N. Damaschke; G. Guesbet; G. Gre'han; H. Mignon; C. Tropea: Applied Optics 37, 10 (1998) 1752-1761

49) A. Doicu; S. Schabel; F. Ebert: Part. Part. Syst. Charac. 13 (1996) 79-88

50) A. Doicu; J. Köser; Th. Wriedt; K. Bauckhage: Part. Part. Syst. Charac. 15 (1998) 257-262

51) Th. Wriedt; K. Bauckhage; A. Schöne: IEEE Transactions on Instrumentation and Measurement Vol. 38 No. 5; Oct. 1989; 984-990

52) G. Göbel; A. Doicu; Th. Wriedt; K. Bauckhage: Part. Part. Syst. Charac. 14 (1997) 283-289

53) A. Doicu; G. Göbel; Th. Wriedt; K. Bauckhage: Part. Part. Syst. Charac. 15 (1998) 225-229

54) J. Tillwick; V. Uhlenwinkel; K. Bauckhage: Int. J of Heat and Fluid Flow 20 (1999) 530-537

55) J. Tillwick: Dissertation Universität Bremen (1999)

56) H. Dahl: Dissertation Universität Bremen (1999)

\section{Author's short biography}

\section{Klaus Bauckhage}

K. Bauckhage is Professor of Chemical Engineering, Faculty of Process Engineering at the University of Bremen (since 1978). Since 1986 he is Director at the Stiftung Institut für Werkstofftechnik, Bremen. After receiving his diploma in Chemical Engineering in 1966 at the Technische Universität Berlin, he was with the FRIEDRICH UHDE GmbH from 1966 to 1968. He received his Dr.-Ing. from the Technische Universität Clausthal in 1973. 